Paediatr Paedolog 2018 $53: 226-230$ https://doi.org/10.1007/s00608-018-0608-z Online publiziert: 10. September 2018 (c) Der/die Autor(en) 2018

CrossMark

\title{
Verletzungsmuster von Kindern und Jugendlichen auf steirischen Skipisten
}

\author{
Auswertung der Unfallzahlen von \\ Skigebieten des steirischen \\ Pistengütesiegels
}

Wintersport hat eine langjährige Tradition in Österreich [1]. Neben dem Aspekt der Erholung und der sportlichen Betätigung für den einzelnen Wintergast stellt der Wintersport einen wichtigen ökonomischen Faktor für die steirischen Skigebiete dar [2]. Aus diesem Grund ist die Prävention und Unfallreduktion ein wichtiger Faktor für die Region. Daher wurde in Kooperation zwischen der Wirtschaftskammer Steiermark, dem Land Steiermark und dem Verein Große Schützen Kleine das Steirische Pistengütesiegel (PGS) gegründet. Ziel des PGS ist die Verbesserung und Kontrolle der Infrastruktur der steirischen Skigebiete hinsichtlich Unfallprävention und Reduktion der Unfallzahlen. In diesem Zusammenhang berichteten die Autoren auch schon über ein deutlich niedrigeres Unfallrisiko in den Gebieten des PGS im internationalen Vergleich [3]. Alle Skigebiete des PGS müssen bei jedem Unfall, der die Anwesenheit des Pistenrettungsdiensts erfordert, einen standardisierten Unfallbogen ausfüllen. Diese Bögen werden am Saisonende zentral gesammelt und archiviert. Ziel dieses Beitrags ist es, aus den Zahlen der Wintersaison 2014/2015 die Unfallzahlen, Verletzungs- und Behandlungsmuster von Kindern und Jugendlichen auszuarbeiten.

\section{Ergebnisse der eigenen Untersuchung}

\section{Allgemeine demographische Daten}

In der betrachteten Wintersaison wurden insgesamt 1844 Pistenunfälle erfasst. Die Auswertung der Daten wurde von der Ethikkommission der Medizinischen Universität Graz genehmigt. In 464 Fällen $(25,1 \%)$ waren dabei Kinder oder Jugendliche (unter dem 18. Lebensjahr) betroffen. Von diesen waren $55,5 \%$ männlich und $44,5 \%$ weiblich. Das mittlere Alter betrug 12,7 Jahre bei männlichen und 12,6 Jahre bei weiblichen Verunfallten $(p=0,644$; t-Test $)$.

Betrachtet man nur Kinder und Jugendliche, so waren die Altersgruppen 12-15 und 15-18 Jahre am häufigsten in Pistenunfälle verwickelt (• Abb. 1). Eine Analyse der Unfallhäufigkeit nach Altersgruppe und Geschlecht ergab keine signifikanten Unterschiede ( $p=0,694$; ChiQuadrat-Test).

\section{Ausgeführte Wintersportart}

Eine Analyse der ausgeführten Wintersportart zeigte - entgegen den Trends in der Vergangenheit - eine Häufung von Verletzungen beim Skifahren $(74,5 \%)$ im Vergleich zum Snowboarden (25,5\%), wobei ein Peak an Snowboardunfällen in den Altersgruppen von $12-15$ und 15-18 Jahren zu sehen war (• Abb. 1). Bei der Art des ausgeführten Wintersports gab es keine signifikanten Unterschiede zwischen den Geschlechtern (Snowboarden bei $28,3 \%$ der männlichen und 22,0\% der weiblichen Verletzten; $p=0,132$; Chi-Quadrat-Test).

\section{Unfallort, Unfallmechanismus, Unfallzeit und Abtransport von der} Piste

Die meisten Unfälle (76,8\%) ereigneten sich in der Pistenmitte. Weitere Häufungen fanden sich am Pistenrand $(6,9 \%)$ und im Fun Park (6,2\%; • Abb. 2). Der häufigste Unfallmechanismus war der Sturz ohne Kollision (89,7\%). Bezüglich des Unfallorts und des Unfallmechanismus wurden keine signifikanten Unterschiede zwischen Skifahrern und Snowboardern gefunden ( $p=0,322$ bzw. $p=0,240$; Chi-Quadrat-Test).

Ein Häufigkeitsgipfel an Pistenunfällen zeigte sich um die Mittagszeit und die frühen Nachmittagsstunden (•Abb. 2), wobei 16,5\% der Unfälle von 8-11 Uhr, 47,3\% zwischen 11:01 und 14:00 und $36,2 \%$ zwischen 14:01 und Betriebsschluss verzeichnet wurden.

Der Abtransport vom Unfallort erfolgte in $40,6 \%$ der Fälle mit dem Schneemobil, in 16,4\% mit dem Akija, in 20,3\% 

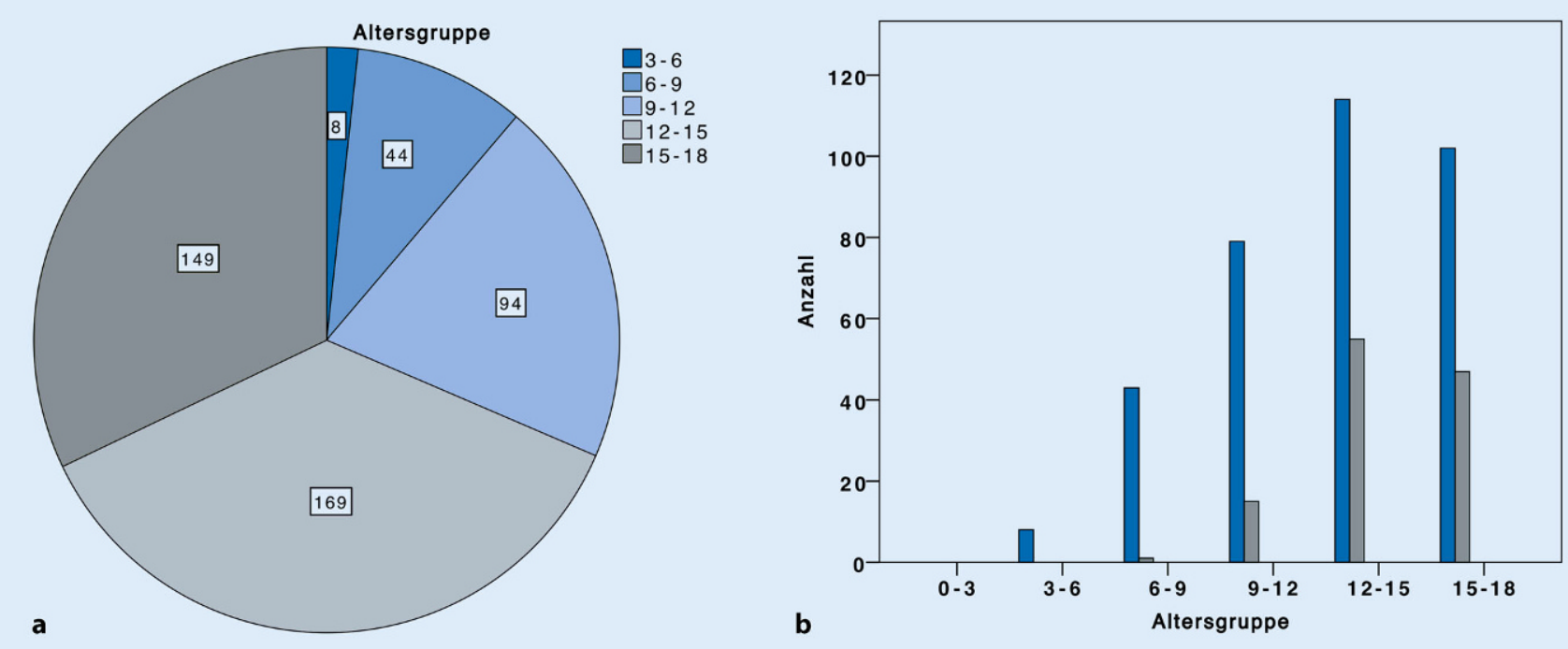

Abb. 1 ॥ Unfallzahlen nach Altersklassen in Jahren (a) und nach Wintersportarten (b, dunkelblau Skifahrer, grau Snowboarder)
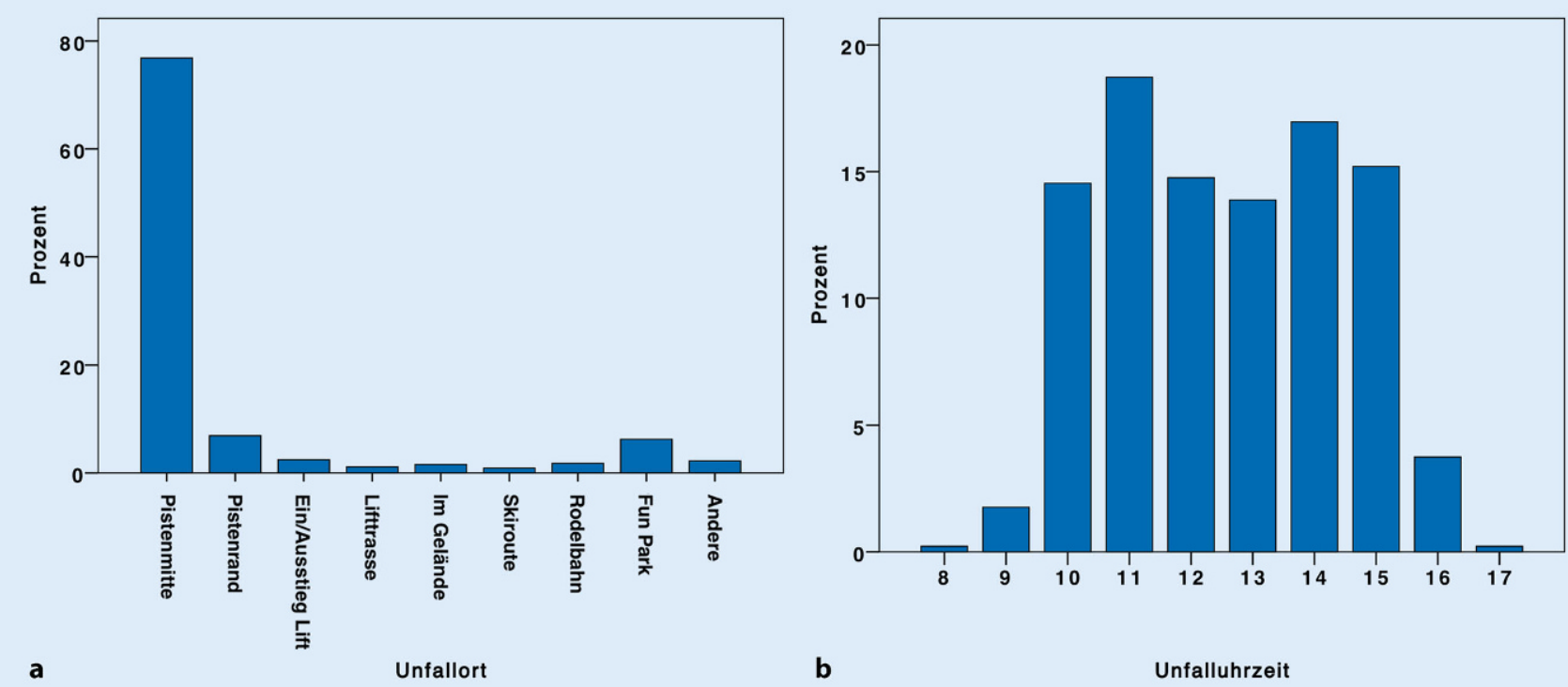

Abb. 2 ^ a Unfallorte, b Unfalluhrzeit

mit dem Skilift und in 9,6\% der Fälle mit dem Notarzthubschrauber.

\section{Verletzungsmuster}

Die nachfolgende Tabelle (• Tab. 1) gibt eine genaue Übersicht über die verletzten Körperregionen. Zusätzlich findet sich eine Darstellung der Unterschiede zwischen Geschlechtern und Wintersportarten.

Insgesamt waren Unterarm, Knie, Unterschenkel und Schulter die am häu- figsten verletzten Körperregionen. Beim Vergleich der verletzten Körperregionen wurde kein signifikanter Unterschied zwischen den Geschlechtern festgestellt. Bezüglich der Wintersportarten fanden sich signifikante Häufungen von Unterarmverletzungen bei Snowboardern sowie Knie- und Unterschenkelverletzungen bei Skifahrern.

\section{Krankenhausdaten}

Von allen verunfallten Kindern und Jugendlichen wurden von 260 (56\%) Krankenhausdaten erhoben. Eine Fraktur wurde bei $52,1 \%$ der Verunfallten diagnostiziert; 77,3\% der Patienten wurden ambulant und 22,7\% stationär versorgt, wobei $44,2 \%$ der Patienten einer operativen Behandlung bedurften. Der durchschnittliche Krankenhausaufenthalt betrug 3,9 Tage (Standardabweichung 3,2 Tage). Die Aufnahme auf 


\section{Castellani $\cdot$ G. Singer $\cdot$ T. Petnehazy $\cdot$ D. Wernitznigg $\cdot$ H. Till}

\section{Verletzungsmuster von Kindern und Jugendlichen auf steirischen Skipisten. Auswertung der Unfallzahlen von Skigebieten des steirischen Pistengütesiegels}

\section{Zusammenfassung}

Wintersport hat eine lange Tradition in Österreich. Neben den Aspekten der Erholung und der sportlichen Betätigung für den einzelnen Wintergast spielt der Wintersport auch eine wichtige ökonomische Rolle. Das Steirische Pistengütesiegel hat die Optimierung infrastruktureller Verhältnisse zur Reduktion der Unfallzahlen in steirischen Skigebieten zum Ziel. Für jeden Unfall, bei dem die Anwesenheit des Pistenrettungsdiensts benötigt wird, wird routinemäßig ein Unfallbogen ausgefüllt. Für diese Studie wurden die Unfallbögen der Saison 2014/2015 mit besonderem Augenmerk auf das Verletzungsmuster von Kindern und Jugendlichen ausgewertet. Zusätzlich wurden - wenn verfügbar - die
Krankenhausdaten der Patienten erhoben. Be 1844 erfassten Pistenunfällen waren 464 Kinder und Jugendliche betroffen. Der häufigste Unfallort war die Pistenmitte, wobei sich die Verletzungen vor allem um die Mittagszeit und in den frühen Nachmittagsstunden ereigneten. Der Großteil der Verletzten wurde mit Schneemobil, Liftanlage oder Akija abtransportiert. In fast 10\% der Unfälle war der Einsatz des Rettungshubschraubers erforderlich. Es zeigten sich Häufungen der Verletzungen im Bereich von Unterarm, Knie, Unterschenkel und Schulter. Bei einer Analyse der Wintersportarten zeigte sich eine Häufung der Unterarmläsionen bei Snowboardern und der Knie- und Unterschen- kelverletzungen bei Skifahrern. Frakturen erlitten 52,1\% aller Verletzten. Eine stationäre Krankenhausbehandlung war bei $22,7 \%$ der Fälle erforderlich, wobei 44,2 \% einen operativen Eingriff benötigten. Hinsichtlich zukünftiger Präventionsstrategien muss vor allem auf die Pistenmitte als Unfallort fokussiert werden. Die Häufung von Knie- und Unterschenkelverletzungen bei Skifahrern sollte zu einer jährlich erforderlichen Kontrolle von Skibindungen und Skischuhen durch Fachbetriebe veranlassen.

Schlüsselwörter Wintersport · Unfallverhütung · Skifahren . Snowboarden · Trauma

\section{Injury pattern of children and adolescents on Styrian ski slopes. Accident analysis of winter sport resorts holding the Styrian Slope Certificate}

\section{Abstract}

Winter sport has a long tradition in Austria. Besides the recuperative and recreational aspects for the individual winter guest, winter sports play an important economic role. The Styrian Slope Certificate aims to improve infrastructural quality in Styrian ski resorts to reduce the number of winter sport accidents. For every accident requiring the presence of the ski patrol, a standardized form is routinely completed. For this study, the forms of the 2014/2015 season were analyzed in detail, specifically focusing on the injury patterns of children and adolescents. Moreover, patients' data at the local hospitals were retrieved if available. Of a total of 1,844 documented skiing accidents, 464 involved children or adolescents. The most common site of accidents was the middle of the ski slopes, where accidents predominantly occurred around noon and in the early afternoon. Most of the injured were transported by snow mobile, lift or rescue sledge. In almost $10 \%$ of the accidents, the use of a rescue helicopter was required. The most commonly injured body regions were the forearm, knee, lower leg, and shoulder. An analysis of types of winter sports showed that snowboarders sustained more injuries of the forearm, and skiers had a predominance of knee and lower leg lesions. Fractures were sustained by $51.2 \%$ of all of those injured. Inpatient treatment was required by $22.7 \%$ of cases, $44.2 \%$ of which needed a surgical intervention. With regard to future prevention strategies, focus should be on the middle of the slope as the most common accident site. Additionally, the high number of knee and lower leg injuries should prompt essential annual checks of binding and ski boots by specialists.

Keywords

Winter sports · Injury prevention · Skiing · Snowbarding $\cdot$ Trauma eine Intensivstation war in $1,2 \%$ der Fälle erforderlich und dauerte im Mittel 4,5 Tage.

\section{Diskussion}

In der betrachteten Saison betrafen etwa ein Viertel der Pistenunfälle in den Gebieten des PGS Kinder und Jugendliche. Da die Gesamtzahl der in den Wintersportregionen anwesenden Sportler nach Alter aufgeschlüsselt nicht bekannt ist, können keine Angaben über das relative Verletzungsrisiko dieser Altersgrup- pe gemacht werden. In der betrachteten Wintersaison lag jedoch das generelle Unfallrisiko (bezogen auf die 1844 erfassten Pistenunfälle) bei 0,74 pro 1000 Skitage [3]. Generell war das Verletzungsrisiko dabei geringer als bei anderen Sportarten wie z. B. dem Ballsport oder anderen Teamsportarten [4].

Generell wurden die meisten Unfälle in der Altersklasse zwischen 12-15 und 15-18 Jahren dokumentiert. Da, wie oben beschrieben, die anteiligen Wintersportgäste in den jeweiligen Altersklassen nicht bekannt sind, kann auch hier kein relatives Verletzungsrisiko abgeleitet werden. Insgesamt dürften jedoch mehr Wintersportgäste mit diesem Alter in den Gebieten unterwegs gewesen sein. Zusätzlich sind eine höhere Risikobereitschaft und ein rasanterer Fahrstil in diesem Alter denkbar. Beide Annahmen würden die höheren Verletzungszahlen in diesen Altersklassen erklären.

Entgegen bisheriger Medienberichte, die Unfallhäufungen am Nachmittag beschrieben, zeigt sich in unserer Analyse eine Häufung der Pistenunfälle um die Mittagszeit und in den frühen Nachmit- 
tagsstunden, wobei die Ursache für diese Verteilung noch ungeklärt ist. Als Unfallort ist nach wie vor die Pistenmitte führend. Auch der Pistenrand, die $\mathrm{Zu}$ und Ausstiege aus den Liftanalgen und die Fun Parks haben sich als neuralgische Stellen erwiesen und werden in den Fokus zukünftiger Präventionsstrategien gerückt werden. Entgegen bisherigen Berichten in der Literatur [5] beobachteten wir jedoch keine Häufung schwerer Verletzungen in Fun Parks.

Über alle Verletzten zeigte sich eine Häufung von Verletzungen im Bereich des Unterarms, Knies, Unterschenkels und der Schulter. Die Verletzungsmuster entsprechen dabei weitgehend Berichten aus der Literatur $[6,7]$. Von diesen Verletzungen betrafen $75 \%$ Skifahrer und $25 \%$ Snowboarder. Diese Diskrepanz dürfte auf einem generellen Rückgang der Snowboarder auf den Pisten begründet liegen.

Bei den Verletzungsmustern fanden sich bei Snowboardern signifikant häufiger Unterarmverletzungen, was auch von anderen Autoren in der Literatur beschrieben wurde $[8,9]$. Bei Skifahrern waren signifikant häufiger Knie und Unterschenkel betroffen. Vor allem über die Häufung von Unterschenkelverletzungen beim Wintersport im Kindesalter wurde schon berichtet $[2,10]$. Die bei Erwachsenen beschriebenen Geschlechterunterschiede im Verletzungsmuster $[11,12]$ konnten in dieser Analyse von Kindern und Jugendlichen nicht nachvollzogen werden.

Aus den ermittelten Daten ergeben sich die folgenden wichtigen Aspekte für zukünftige Präventionsstrategien: die Pistenmitte ist nach wie vor der bei Weitem häufigste Unfallort. Vor Aufenthalten in der Pistenmitte sollte - insbesondere an unübersichtlichen Stellen Abstand genommen werden. Auch der Pistenrand und die Liftzu- und -ausstiege wurden als neuralgische Stellen identifiziert. Hier sollte zur Unfallvermeidung vor allem auf die Beachtung der Skiregeln der Fédération Internationale de Ski (FIS) geachtet werden. Auf die Wichtigkeit der Schutzausrüstung wurde bereits in unserem vorangegangenen Beitrag hingewiesen [3]. Hinsichtlich der Unterschenkel- und Knieverletzungen kann nur immer wieder auf eine Fachberatung beim Kauf der Ausrüstung und auf eine regelmäßige (jährliche) Wartung der Bindungen durch entsprechende Fachbetriebe hingewiesen werden. Vor allem in Phasen des starken Wachstums verändern sich anthropometrische Daten schnell, was eine neuerliche Einstellung der Bindung wegen veränderter Größe und Gewicht erfordert. Zuletzt sollte - für den Fall einer Verletzung - auf die Wichtigkeit eines adäquaten Versicherungsschutzes für die ganze Familie hingewiesen werden. Dies betrifft nicht notwendigerweise nur die Krankenhausversorgung, sondern auch die Abdeckung von Schäden bei anderen Kosten für Bergung, Rechtsberatung und Gerichtskosten.

\section{Hier steht eine Anzeige.}

\section{Springer}


Tab. 1 Übersicht über die Verletzungsmuster. Die Geschlechterverteilung gibt den relativen Anteil des Geschlechts an der jeweils verletzten Region

\begin{tabular}{|c|c|c|c|c|c|c|c|}
\hline Region & Gesamt (\%) & $\begin{array}{l}\text { Männlich } \\
\text { (\%) }\end{array}$ & $\begin{array}{l}\text { Weiblich } \\
\text { (\%) }\end{array}$ & $p$ & Ski (\%) & $\begin{array}{l}\text { Snowboard } \\
(\%)\end{array}$ & $p$ \\
\hline Kopf & 6,9 & 51,6 & 48,4 & 0,851 & 12,9 & 8,8 & 0,513 \\
\hline Halswirbelsäule & 1,1 & 0 & 100 & 0,101 & 1,5 & 0 & 0,570 \\
\hline Brustwirbelsäule/Lendenwirbelsäule & 4,5 & 58,3 & 41,7 & 0,775 & 4,6 & 4,4 & 1,0 \\
\hline Thorax & 3,0 & 50,0 & 50,0 & 1,0 & 4,1 & 0 & 0,116 \\
\hline Abdomen & 0,4 & 100 & 0 & 1,0 & 0,5 & 0 & 1,0 \\
\hline Becken & 2,6 & 85,7 & 14,3 & 0,126 & 2,5 & 2,9 & 1,0 \\
\hline Schulter & 10,2 & 69,2 & 30,8 & 0,100 & 7,7 & 16,1 & 0,059 \\
\hline Oberarm & 3,4 & 66,7 & 33,3 & 0,509 & 3,1 & 4,4 & 0,700 \\
\hline Unterarm & 23,0 & 55,0 & 45,0 & 0,770 & 10,8 & 57,3 & $<0,001$ \\
\hline Hand & 4,2 & 45,4 & 54,6 & 0,760 & 3,6 & 5,8 & 0,484 \\
\hline Oberschenkel & 5,7 & 46,6 & 53,4 & 0,791 & 6,2 & 2,9 & 0,369 \\
\hline Knie & 21,1 & 49,0 & 51,0 & 0,544 & 26,4 & 5,8 & $<0,001$ \\
\hline Unterschenkel & 18,5 & 44,8 & 55,2 & 0,207 & 23,8 & 4,4 & $<0,001$ \\
\hline Fuß & 0,2 & 100 & 0 & 1,0 & 0 & 1,4 & 0,254 \\
\hline Mehrere Regionen & 4,6 & 54,5 & 45,5 & 1,0 & 8,2 & 8,8 & 0,805 \\
\hline
\end{tabular}

Bei den Wintersportarten beziehen sich die Prozentangaben auf die in der jeweiligen Sportart insgesamt verunfallten Personen Kursiv häufigste Körperregionen, fett signifikante Unterschiede

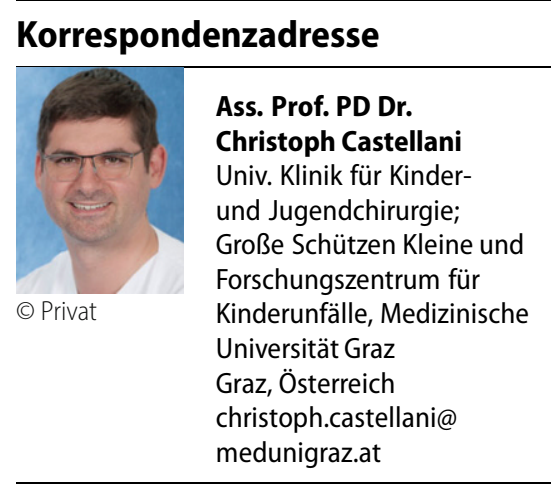

Ass. Prof. PD Dr. Christoph Castellani ist Facharzt für Kinder- und Jugendchirurgie an der Univ.-Klinik für Kinder- und Jugendchirurgie der Medizinischen Universität Graz. Er ist Vizepräsident des Vereins Große Schützen Kleine und Mitglied der Kommission des Steirischen Pistengütesiegels.

Danksagung. Diese Untersuchung wurde von Große Schützen Kleine und dem Forschungszentrum für Kinderunfälle unterstützt.

Funding. Open access funding provided by Medical University of Graz.

\section{Einhaltung ethischer Richtlinien}

Interessenkonflikt. C. Castellani, G. Singer, T. Petnehazy, D. Wernitznigg und $\mathrm{H}$. Till geben an, dass kein Interessenkonflikt besteht.

Alle beschriebenen Untersuchungen am Menschen wurden mit Zustimmung der zuständigen EthikKommission, im Einklang mit nationalem Recht sowie gemäß der Deklaration von Helsinki von 1975 (in der aktuellen, überarbeiteten Fassung) durchgeführt.

Open Access. Dieser Artikel wird unter der Creative Commons Namensnennung 4.0 International Lizenz (http://creativecommons.org/licenses/by/4.0/deed. de) veröffentlicht, welche die Nutzung, Vervielfältigung, Bearbeitung, Verbreitung und Wiedergabe in jeglichem Medium und Format erlaubt, sofern Sie den/die ursprünglichen Autor(en) und die Quelle ordnungsgemäßnennen, einen Linkzur Creative Commons Lizenz beifügen und angeben, ob Änderungen vorgenommen wurden.

\section{Literatur}

1. Burtscher M, Ponchia A (2010) The risk of cardiovascular events during leisure time activities at altitude. Prog Cardiovasc Dis 52(6):507-511

2. Ruedl G et al (2014) Current incidence of accidents on Austrian ski slopes. Sportverletz Sportschaden 28(4):183-187

3. Castellani C et al (2017) Wer Köpfchen hat, der schützt es. Padiatr Padol. https://doi.org/10.1007/ s00608-017-0456-2

4. Pons-Villanueva J, Segui-Gomez M, MartinezGonzalez MA (2010) Risk of injury according to participation in specific physical activities: a 6 -year follow-up of 14356 participants of the SUN cohort. Int JEpidemiol 39(2):580-587

5. Goulet $C$ et al (2007) Risk factors associated with serious ski patrol-reported injuries sustained by skiers and snowboarders in snow-parks and on other slopes. Can J Public Health 98(5):402-406

6. Aschauer E et al (2007) Injuries and injury risk in skiing and snowboarding. Unfallchirurg 110(4):301-306

7. Ruedl $G$ et al (2009) Distribution of injury mechanisms and related factors in ACL-injured female carving skiers. Knee Surg Sports Traumatol Arthrosc 17(11):1393-1398
8. Ehrnthaller C, Gebhard F, Kusche H (2014) Typical injuries in snowboarding. Possible prevention strategies. Unfallchirurg 117(1):7-12

9. Kim S et al (2012) Snowboarding injuries: trends over time and comparisons with alpine skiing injuries. Am J Sports Med 40(4):770-776

10. Schulz D (2012) Unfälle und Verletzungen im alpinen Skisport. Zahlen und Trends 2011/2012 https://www.ski-online.de/stiftung-sicherheit/ projekte/detail/asu-unfallanalyse-290.html. Zugegriffen: 10. Nov. 2016

11. Ekeland A, Rodven A (2010) Skiing and boarding injuries on Norwegian slopes during two winter seasons. J ASTM Int 7(4):1-8

12. Burtscher $M$ et al (2008) Effects of modern ski equipment on the overall injury rate and the pattern of injury location in Alpine skiing. Clin Sport Med 18(4):355-357 